\title{
Photodarkening-Induced Phase Distortions And Their Effects in Single-Channel And Coherently Combined Yb-Doped Fiber Chirped Pulse Amplification Systems
}

\author{
Yujun Feng, Betty Meng Zhang and Johan Nilsson
}

\begin{abstract}
We use numerical simulations to investigate the effects of photodarkening (PD) on pulse compression and coherent combination following chirped-pulse amplification in Yb-doped fiber amplifiers. Even though we increase the pump power to keep the pulse energy constant, PD degrades the pulses due to phase distortions resulting from heating acting already within a pulse as well as to increased self-phase modulation if the fiber length is optimized for the PD-free fiber at beginning of life. By contrast, in a fiber shorter than this, the drop in peak power is a relatively modest $35 \%$ even at a PD propagation loss as large as $5 \mathrm{~dB}$, thus providing effective mitigation against PD-induced phase distortions. The improvements extend to the combination efficiency of beam-combined systems.
\end{abstract}

Index Terms - Fiber lasers, Ultrafast optics, Phase distortion

\section{INTRODUCTION}

$\mathrm{P}$ HOTODARKENING [1]-[11] (PD) can degrade fiber lasers by gradually increasing the losses of light propagating in the core, and can be viewed as an aging effect that limits the system lifetime. In a Yb-doped fiber (YDF), this reduces the output power and pulse energy. To the extent that PD cannot be eliminated, a common remedy is then to increase the pump power to compensate for the PD loss, but since the scope for this is limited, PD can still limit the lifetime of fiber laser systems [12]. Furthermore, PD also increases the thermal load, which can also limit the lifetime as well as the achievable performance, e.g., through the interplay between PD and thermal mode instability (TMI) [13]. TMI depends on the relations between the optical phase of interacting modes and a thermally driven grating [14]. Also other systems depend on the optical phase in different ways [15], [16], and may therefore be degraded by the effect of PD on the phase [5]. This includes chirped-pulse amplification (CPA) systems for ultrashort pulses [16], [17]. In these, the heat generated within a pulse can be enough to distort the phase during the pulse, on timescales that are too short for heat conduction to be significant [18]-[21].

Manuscript received xx xx 2018; revised xx xx, 2018; accepted xx xx, 2018. Date of publication xx xx, 2018; date of current version xx xx, 2018. This work was supported in part by the Air Force Office of Scientific Research (AFOSR) (FA9550-14-1-0382). (Corresponding author: Johan Nilsson. Email: jn@orc.soton.ac.uk)
The heat arises from the quantum defect (QD), but PD adds to this [13]. Photodarkening may then not only reduce the pulse energy, but also distort the phase and thus degrade the quality of the compressed pulses. A further complication is the presence of self-phase-modulation (SPM). Short fibers can be used to reduce SPM, but these require high Yb-concentrations, which are more prone to PD. This creates a tradeoff between the desire to use shorter high-concentration fibers to reduce the SPM, and the use of longer lower-concentration fibers to reduce PD. The optimum tradeoff depends on the operating conditions. For example, to achieve high gain as well as the energy storage required for high-energy pulses in a short fiber, the $\mathrm{Yb}$ inversion is often high. This accelerates PD [6]. Even in cases when it is possible to increase the pump power to compensate for the loss in output energy, the phase distortions cannot be easily compensated. Furthermore, if the energy loss is compensated then the evolution of the energy along the fiber, and thus the amount of SPM, change. These factors make PD a particular concern for fiber CPA systems.

Coherently combined multi-channel amplifier systems also depend on the optical phase. This includes coherent beam combination (CBC) of femtosecond pulses [22]-[24]. The number of concepts and experimental demonstrations is increasing rapidly (e.g., [17], [25]-[29]), and femtosecond CBC systems are also commercially available [30]. Whereas the phase control loops essential to actively phased CBC will automatically compensate for slow phase drifts, they cannot compensate for fast intra-pulse phase distortions. Such distortions will degrade $\mathrm{CBC}$ of pulses insofar as they vary between channels. This is especially relevant for massively parallel systems, proposed as drivers for particle accelerators [31]. Demands for overall reliability and ease of maintenance of such systems translate into exceptional requirements on reliability and resilience to degradation of individual channels. There are plausible scenarios in which the photodarkening varies considerably between channels. For example, in a small system it may be possible to replace all fibers if some of them

Yujun Feng, Betty Meng Zhang and Johan Nilsson are with the Optoelectronics Research Centre, University of Southampton, Southampton SO17 1BJ, UK 
degrade in order to maintain channel uniformity, but this may not be realistic in a high-count system. Then, the PD loss may be small in renewed channels, and high in channels closer to end of life. Similarly, whereas a low-count system can be based on fibers from a single YDF preform, a large-count system would require fibers from several preforms, so fiber-to-fiber variations in PD-characteristics are likely to be higher.

In this paper, we use simulations to investigate to what extent PD can distort the phase and thus degrade the pulse compression in YDF CPA systems. We also investigate how differences in PD between arms affect the combination efficiency of a simple two-channel system and discuss the impact on high-count systems. We treat the case when the pump power is adjusted to keep the pulse energy constant and find that in a system optimized for beginning of life, the direct PD heating as well as the change in the SPM can both lead to distortions of several radians and thus significantly degrade the system. However, if the fiber is shortened to better reflect anticipated worst-case end-of-life conditions then the phase distortions can be acceptable even at $5 \mathrm{~dB}$ of total PD propagation loss. These effects occur already at low average power. Other effects may occur at high average powers as a result of PD and increased thermal load, e.g., increased thermal mode squeezing [32] which also increases the SPM. However, these are not included in our simulations.

\section{METHOD}

Fig. 1 illustrates the CPA system with two-channel CBC, and also the single-channel subset. We used RP Fiber Power for the simulations of fiber propagation and amplification. This uses a split-step algorithm which accounts for chromatic dispersion, $\mathrm{SPM}$, wavelength-dependent $\mathrm{Yb}$ gain, and the spectral distortion of chirped pulses in the presence of intra-pulse gain saturation[33].

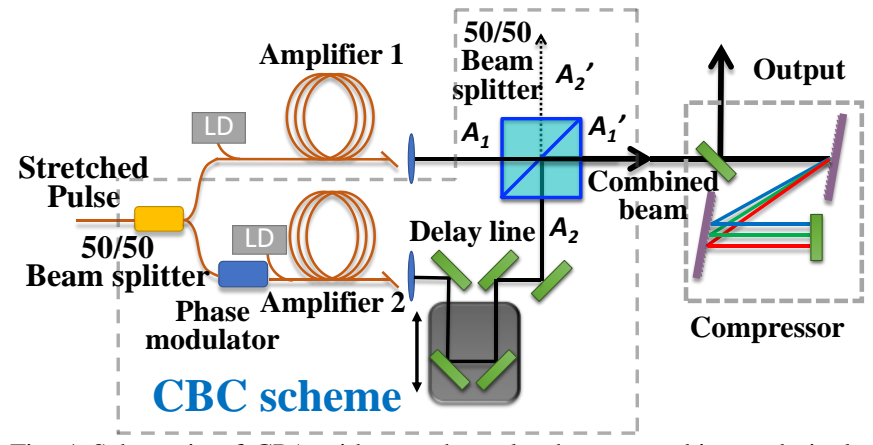

Fig. 1 Schematic of CPA with two-channel coherent combing and singlechannel subset. The phase modulator optimizes the average phase but cannot compensate for intra-pulse phase variations.

Using the scripting capabilities of the software [34], we also introduced intra-pulse phase distortions caused by heating. Heating and the resulting temperature change can be divided into a typically-dominant slow response which depends on the average power and heat conduction [35], and a fast transient response [18], [21]. They both affect the refractive index through the thermo-optic effect, and thus the phase, and they both have memory, but the slow effect cannot respond within a single pulse. Therefore, it does not distort the all-important phase envelope of a pulse and is not included in our simulations.
On the other hand, even a single pulse can generate significant heat. As it comes to QD-heating, this can be divided into a pumping part and a stimulated-emission part. The pumping part is the difference between the pump photon energy and the average change of energy of an ion as it becomes excited into the upper laser level. However, the pump energy deposited during the typical duration of a chirped pulse (e.g., $1 \mathrm{~ns}$ ) is small, so the pumping part of the QD can be neglected. As it comes to stimulated emission, this terminates in the ground state in $\mathrm{Yb}$, so there is no subsequent inter-band relaxation. However, it still leads to QD-heating through intra-band relaxations between Stark sub-levels and interactions with glass matrix vibrations (phonons), so that a Boltzmann distribution is maintained between the Stark levels in the upper laser level as well as in the terminal level. Thus, the QD heating induced by stimulated emission is the difference between the average change of energy of an $\mathrm{Yb}$-ion as it is de-excited and the signal photon energy. In $\mathrm{Yb}$, this is a small fraction of the signal photon energy, e.g., $5 \%-10 \%$. As it comes to PD, the fraction of the signal pulse energy that it absorbs is all converted to heat.

We treat both the PD and the QD-heating as being instantaneous. As it comes to the QD-heating, the energy gap between Stark levels can be bridged by a single phonon, with time constant on the ps or sub-ps scale [36], [37]. After this timescale, the deposited QD-energy is (locally) in thermal equilibrium, and its effect on the phase of a propagating pulse is determined by the local temperature and the thermo-optic coefficient $d n / d T$. We also expect that the deposited energy can affect the phase during the short time before it reaches thermal equilibrium. The details of this are not known, but it is neglected on account of the short duration relative to the pulse.

The fast excitation dynamics (not to be confused with the growth characteristics) of the photodarkening are not known to us, and may well vary between defect centers of different types. Centers with relatively long-lived excited-state lifetimes are possible, e.g., quenched $\mathrm{Yb}^{3+}$-ions, which have been associated with PD in YDFs [2], [9]-[11]. These could trap the energy for the duration of the signal pulse, and thus not contribute instantaneously to the heating. However experimental evidence that the energy lost to PD is absorbed by such long-lived centers is lacking. On the other hand, time constants for defect-induced thermal damage in silica are of the order of $10 \mathrm{ps}$ or less [38]. Therefore we consider the PD centers to relax and transfer the energy to the lattice through longitudinal phonon scattering [37], with timescale again typically $10 \mathrm{ps}$, so we treat also the PD-heating as being instantaneous. We note also that it is straightforward to modify our approach if only a fraction of the heating is instantaneous.

In principle, heat conduction can also be a factor in the dynamics of the heating. However, the pulse is too short for heat conduction to be significant during a pulse, which instead occurs between pulses. The heat and temperature thus build up and change the refractive index during a single pulse. The phase distortion caused by the heating transients in segment $m, \Phi_{h}^{m}(t)$ can be calculated by the following equation [18]:

$$
\Phi_{h}^{m}(t)=\frac{2 \pi}{\lambda(t)} \cdot \frac{d n}{d T} \cdot \frac{Q(t)}{A_{e f f, h} \rho C_{v}}
$$


where $\lambda$ is the wavelength, $d n / d T=1.06 \times 10^{-5} \mathrm{~K}^{-1}, A_{\text {eff, }} h$ is the effective area for the instantaneous thermal phase shift [18], $\rho$ $\left(2.70 \times 10^{3} \mathrm{~kg} / \mathrm{m}^{3}\right)$ is the core density, $C_{v}\left(700 \mathrm{~J} \mathrm{~K}^{-1} \cdot \mathrm{kg}^{-1}\right)$ is the specific heat of the core and $Q(t)$ is the heat induced by the pulse in the segment up to time $t$. According to Eq. (1), the thermal phase distortion induced directly by a given amount of heat is length-independent. Well-controlled experiments on the thermal phase response show excellent agreement with this equation for narrow-linewidth pulses in the sub- $\mu$ s regime [21].

With chirped pulses in a YDF, the heating is given by

$Q(t)=\int_{0}^{t} P_{S}^{m}(\tau)\left[g^{m}(\tau)\left(\frac{v_{0}}{v_{s}(\tau)}-1\right)+\alpha_{P D}^{m} \frac{v_{0}}{v_{S}(\tau)}\right] d \tau$

Here, $\alpha_{P D}^{m}$ represents the attenuation caused by PD, $P_{s}^{m}(\tau)$ is the signal power, and $g^{m}(\tau)$ is the net gain in segment $m$. Furthermore, $v_{s}(\tau)$ is the instantaneous signal frequency, and $v_{0}$ corresponds to the average energy difference between the excited state and ground state of $\mathrm{Yb}$-ions Boltzmann-distributed over the Stark levels. Therefore, the first part in the integral represents QD heating in the absence of PD, while the second part represents the heating caused by PD directly, as well as the increase in QD heating resulting from the increase in stimulated emission to compensate for the PD loss. The phase change induced locally by PD and QD heating is added to the numerical propagation according to Eq. (1).

We simulated a forward cladding-pumped fiber amplifier with a $40-\mu \mathrm{m}$ diameter, $0.03-\mathrm{NA}, \mathrm{Yb}$-doped aluminosilicate core and a $200-\mu \mathrm{m}$ diameter inner cladding. The amplifier operates on the fundamental mode, which has an effective area for SPM of $760 \mu \mathrm{m}^{2}$. According to Eq. 43 in Davis's paper [18], the effective area $A_{\text {eff, } h}$ for the instantaneous thermal phase shift becomes $742 \mu \mathrm{m}^{2}$. The core's time constant for thermal conduction becomes $1.0 \mu \mathrm{s}$ [18], which confirms that heat diffusion in $1 \mathrm{~ns}$ is negligible. The amplifier was seeded with positively chirped Gaussian pulses, stretched to $1 \mathrm{~ns}$ and centered at $1045 \mathrm{~nm}$ with $7.8 \mathrm{~nm}$ bandwidth (full width at half maximum, FWHM). This corresponds to a transform-limited Gaussian pulse with duration of 183 fs (FWHM). The saturation energy becomes $0.4 \mathrm{~mJ}$. The repetition rate was $16 \mathrm{kHz}$ and the seed energy $1 \mu \mathrm{J}$. With $5.2 \mathrm{~W}$ pumping at $975 \mathrm{~nm}$, the output signal energy was around $100 \mu \mathrm{J}$ in the absence of PD. For simplicity, we neglect spatial variations of the PD that are expected due to the variations in the $\mathrm{Yb}$ inversion and signal power, as well as the spectral dependence of the PD loss. Since the pump propagates largely in the PD-free cladding, we also neglect the direct losses of pump to PD, which otherwise increase the required pump power and slightly modify the evolution of the signal pulse energy.

In order to focus on induced phase distortions, we maintained this signal energy by pumping harder, as PD was introduced. Note first that the average phase $\varphi$ and group delay $d \varphi / d \omega$ are controlled in CBC and do not matter for the pulse compression. As it comes to remaining, higher-order, intra-pulse phase distortions, it may be possible to adjust the pulse compression or stretching to compensate for these and thus improve the compressed pulse. Although this is cumbersome, it may still be motivated in expensive $\mathrm{CBC}$ systems, but we did not investigate this possibility. Furthermore, even with such adjustments, the combination efficiency still degrades if the PD is different in different channels. For $\mathrm{CBC}$, we assumed that one channel has photodarkened while the other remains pristine. This maximizes the difference between channels, which is the worst case for the combination efficiency.

\section{RESULTS}

Fig. 2 shows how the pulse energy and the phase distortions caused by SPM and thermal effects evolve along a 2-m-long fiber with $\mathrm{Yb}^{3+}$-ion density $3.26 \times 10^{25} \mathrm{~m}^{-3}$ for different levels of total PD propagation loss. These parameters minimize the required pump power $(5.2 \mathrm{~W})$ for $100 \mu \mathrm{J}$ output energy, in the absence of PD. When the PD increases, the energy is maintained at $100 \mu \mathrm{J}$, by increasing the pump power gradually, up to $9.6 \mathrm{~W}$ for 5 -dB PD loss. When the loss gets as high as 5 $\mathrm{dB}$, the heat deposited by a single pulse reaches $80 \mu \mathrm{J}$. We also see that the fiber is now overlength. This affects the B-integral, which quantifies SPM. Without PD, it becomes $20.9 \mathrm{rad}$. At 5 dB PD loss, it grows to $25.9 \mathrm{rad}$. The total thermal phase $\Phi_{h}$ induced directly by the heating is given by Eq. (1) summed over all fiber segments. This is shown in Fig. 2 (b) for the trailing pulse edge, where it is largest, since the thermal phase increases during the pulse. SPM dominates the phase response, but the thermal phase and the SPM both change by several radians, which cannot be neglected. By contrast, the thermal phase from QD is only $0.3 \mathrm{rad}(\lambda / 20)$ in the absence of PD, for $99 \mu \mathrm{J}$ of energy extracted from the Yb-ions, of which $7.2 \mu \mathrm{J}(7.3 \%)$ is converted into heat. For $5 \mathrm{~dB}$ of PD, the energy extracted from the $\mathrm{Yb}$-ions changes to $179 \mu \mathrm{J}$. The thermal phase from QD then increases in proportion, but is at $\sim 0.6$ rad still relatively small.

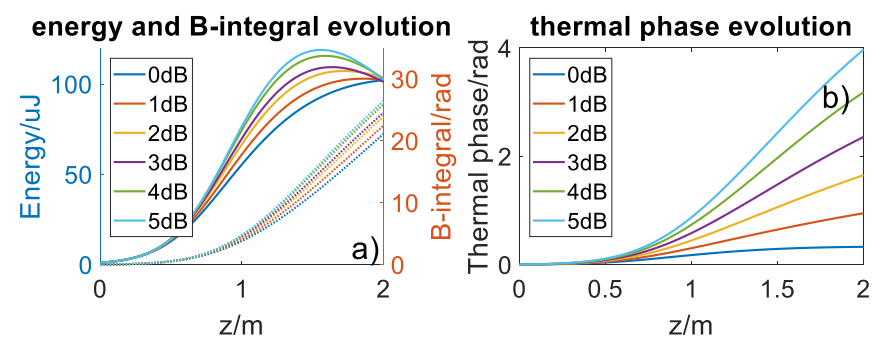

Fig. 2 (a) Signal energy and accumulated B-integral and (b) total accumulated thermal phase as induced in the trailing edge of the pulse in 2-m fiber amplifier for different total PD propagation loss.

We next consider a 1-m fiber with twice the $\mathrm{Yb}$ concentration $\left(6.52 \times 10^{25} \mathrm{~m}^{-3}\right)$ and twice the PD loss per unit length. As expected, the evolution of energy and thermal phase is the same as in Fig. 2, but over $1 \mathrm{~m}$ instead of $2 \mathrm{~m}$, whereas the B-integral drops by half. Fig. 3 shows the total output-pulse phase distortions relative to the PD-free case for different levels of PD for 2-m and 1-m lengths. At $80 \mu \mathrm{J}$ of heating (for $5 \mathrm{~dB}$ of total PD), the change in total thermal phase for both fibers is around $4 \mathrm{rad}$, which agrees well with simple estimates as well as with Fig. 2. However, in the center of the pulse, the distortions differ since there is more SPM in $2 \mathrm{~m}$. The maximum phase change is $\sim 7 \mathrm{rad}(2 \mathrm{~m}$ fiber) and $\sim 5 \mathrm{rad}(1 \mathrm{~m})$.

The phase distortions in Fig. 3 can be partly compensated by a constant slope $d \varphi / d t$. In our linear-chirp regime, this corresponds to a constant $d \varphi / d \omega$. This in turn corresponds to a 
change in the group delay, which is inconsequential in the systems we consider. Nevertheless, the effect of the phase distortions on the pulse compression is still substantial. This is shown in Fig. 4 for a single-channel system. The compression parameters are adjusted to reach the transform-limit for the PDfree case and then kept fixed as the PD increases. With $5 \mathrm{~dB}$ total PD loss, the peak power drops by nearly $80 \%(7 \mathrm{~dB})$ with the 2-m fiber even though the pump power is increased to keep the energy constant. A smaller B-integral improves the compression, but the peak power of the $1 \mathrm{~m}$ fiber amplifier still drops by $\sim 60 \%$ ( $4 \mathrm{~dB})$. In addition, a pre-pulse appears. For comparison, we also considered a case with only thermal phase distortions (i.e., without SPM). Then, the peak power dropped by $\sim 20 \%(1 \mathrm{~dB})$ for $5 \mathrm{~dB}$ PD-loss.
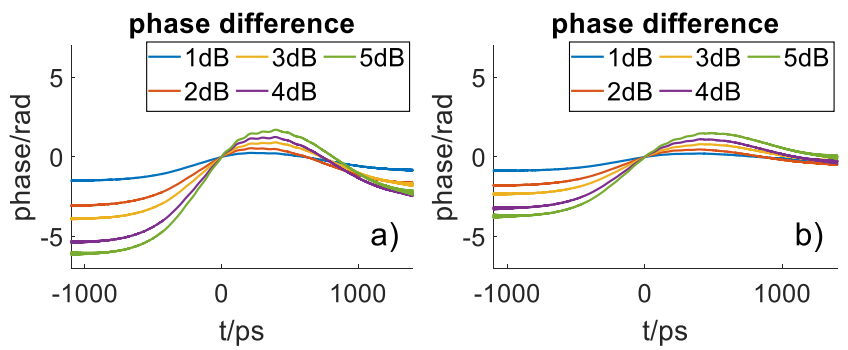

Fig. 3 Change in phase from the PD-free case for different total PD propagation loss in a fiber amplifier of length (a) $2 \mathrm{~m}$ and (b) $1 \mathrm{~m}$.
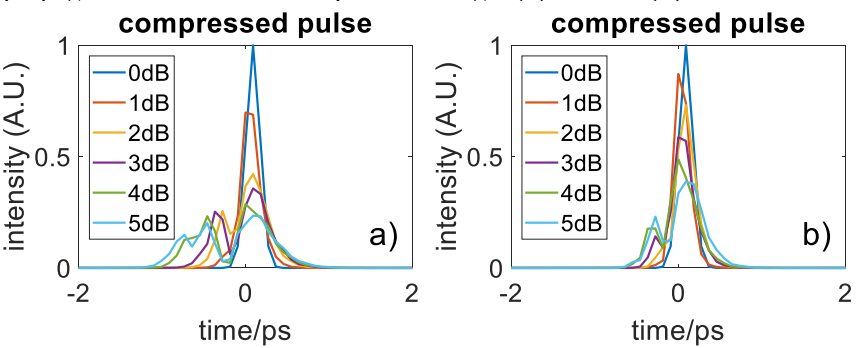

Fig. 4 Compressed pulses from the 2-m (a) and 1-m (b) fiber amplifier at different total $\mathrm{PD}$ propagation loss.

To evaluate $\mathrm{CBC}$, the input seed pulse is divided into two channels with the same energy $(1 \mu \mathrm{J})$, amplified in separate fibers to $100 \mu \mathrm{J}$ per channel, and recombined by a $50 / 50$ beam splitter (Fig. 1). In one arm, the group delay is controlled by an optical delay line and the average phase by a phase modulator. The complex amplitudes in the two output ports of the beam splitter $A_{1}{ }^{\prime}(t)$ and $A_{2}{ }^{\prime}(t)$ are given by

$\left[\begin{array}{l}A_{1}^{\prime}(t) \\ A_{2}^{\prime}(t)\end{array}\right]=\frac{1}{\sqrt{2}}\left[\begin{array}{ll}i & 1 \\ 1 & i\end{array}\right]\left[\begin{array}{l}A_{1}(t) \\ A_{2}(t)\end{array}\right]$

where $A_{i}(t)$ are the input amplitudes. After compensating the group delay between the two pulses, we adjust the phase modulator to maximize the combination efficiency $\eta=$ $\int\left|A_{1}^{\prime}(t)\right|^{2} d t / \int\left|A_{1}(t)\right|^{2} d t+\int\left|A_{2}(t)\right|^{2} d t$. The combined pulse is then compressed with parameters optimized for the PDfree system. Note that mathematically, the order of combination and compression is irrelevant.

Fig. 5 shows pulse shapes following combination and compression. At $5 \mathrm{~dB}$ total PD, the peak power drops by $47 \%$ for the $2-\mathrm{m}$ fiber and by $34 \%$ for the $1-\mathrm{m}$ fiber. A pre-pulse appears, too, but the distortions in shape are smaller than for single-channel pulses in Fig. 4, even if we compare $4 \mathrm{~dB}$ of PD in the $\mathrm{CBC}$ system with $2 \mathrm{~dB}$ in the single-channel system to take into account that the PD of the combined system only affects one channel. Thus, the combination cleans the pulse, but that is at the expense of combining losses, which become $22 \%$ $(2 \mathrm{~m})$ and $11 \%(1 \mathrm{~m})$. The conversion efficiency from pump to combined signal power drops from $31 \%$ without PD to $24 \%$ (2 $\mathrm{m})$ and $27 \%(1 \mathrm{~m})$ with $5 \mathrm{~dB}$ PD.
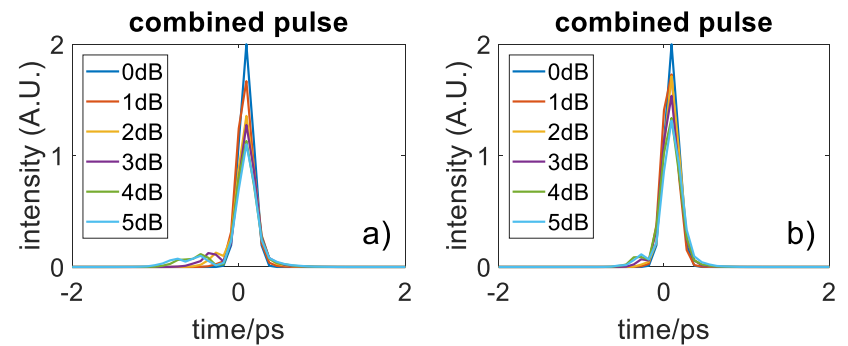

Fig. 5 Pulses from CBC system with different amounts of total PD propagation loss in one channel only. (a) $2 \mathrm{~m}$ fiber (b) $1 \mathrm{~m}$ fiber.

According to our calculations, PD significantly degrades both pulse shape and combination efficiency. However, the lengths of $1 \mathrm{~m}$ and $2 \mathrm{~m}$ were both optimized for highest conversion efficiency without any PD, i.e., at the beginning of life of a system. Fig. 2, for the 2-m fiber, shows that with PD, the fiber becomes too long, which increases the required pump power and the phase distortions. We next instead consider a fiber length optimized for highest conversion efficiency at $5 \mathrm{~dB}$ of total PD propagation loss. The length is $1.5 \mathrm{~m}$, and the $\mathrm{Yb}$ concentration is the same as for the $2-\mathrm{m}$ fiber used before. Fig. 6 shows the results. With $100 \mu \mathrm{J}$ output signal energy as before, the required pump power increases from $5.5 \mathrm{~W}$ to $10.1 \mathrm{~W}$, as the total PD propagation loss increases from $0 \mathrm{~dB}$ to $5 \mathrm{~dB}$. The pump powers are $5.0 \%-6.6 \%$ higher than for the $2-\mathrm{m}$ fiber.
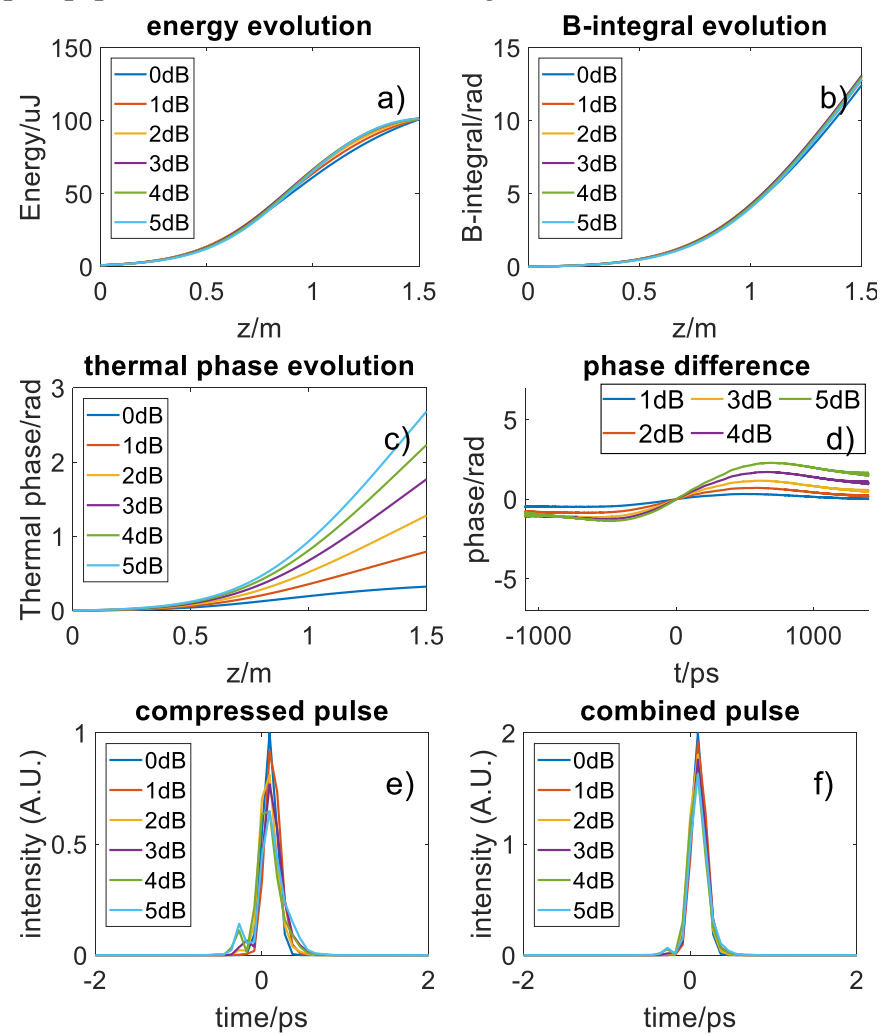

Fig. 6. Results of fiber amplifier shortened to $1.5 \mathrm{~m}$ with different PD losses. Evolution along the fiber of (a) energy, (b) B-integral, and (c) thermally induced phase difference between trailing and leading pulse edge. (d) phase change from PD-free case (e) compressed pulses from single channel (f) compressed pulses for two combined channels. 
The power penalty is modest, and furthermore is for higher PDloss per unit length. By contrast, at equal loss per unit length, the 1.5-m fiber becomes more efficient for sufficiently high PD loss per unit length.

Fig. 6 (a) shows that the energy evolution depends much less on PD for the 1.5-m fiber. Thus, the B-integral only changes from $12.4 \mathrm{rad}$ to $13.0 \mathrm{rad}$ for different PD-levels. The total heat with 5 -dB PD loss decreases to $55 \mu \mathrm{J}$, from $80 \mu \mathrm{J}$ for the fibers optimized for beginning of life. The compressed pulse from a single channel is close to transform-limited even with $5 \mathrm{~dB}$ PD loss, and the peak power remains as high as $70 \%$, compared to $20 \%$ for the $2-\mathrm{m}$ fiber and $40 \%$ for the $1-\mathrm{m}$ fiber. CBC results in quite clean pulses. The peak power drops to $\sim 70 \%$ compared to $50 \%$ for the 2 -m fiber.
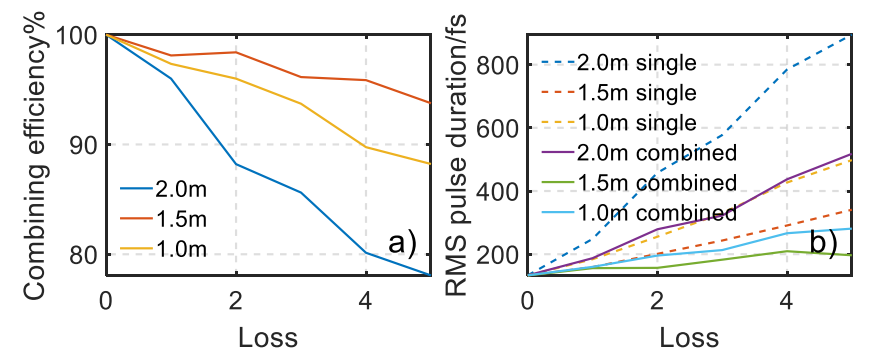

Fig. 7 (a) Combining efficiency and (b) RMS pulse duration of single channel and $\mathrm{CBC}$ systems for different total PD propagation losses for different fibers. The $\mathrm{Yb}$-concentration is the same in the 2-m and $1.5-\mathrm{m}$ fibers, and twice as high in the 1-m fiber.

Fig. 7 shows the combining efficiency and the RMS pulse duration of single-channel and combined pulses, for the three studied fibers. For the 1.5-m fiber optimized for 5-dB of PD, the combining efficiency can be above $94 \%$. By contrast, according to straight-line fits to the efficiency-curves in Fig. 7 (a), the 1-m fiber degrades at a rate which is approximately twice as high, and the 2-m fiber at a rate which is nearly four times as high. Similarly, for the 1.5-m fiber, the duration of the combined pulse increases no more than a few tens of femtoseconds even with $5 \mathrm{~dB}$ of total PD loss. The fibers optimized for beginning-of-life fare much worse. Thus, starting from smaller than $200 \mathrm{fs}$, in the single-channel system the pulse duration grows to $900 \mathrm{fs}$ for $2 \mathrm{~m}$ and $500 \mathrm{fs}$ for $1 \mathrm{~m}$ fiber length. Even though CBC partly cleans the pulse, the duration of the combined pulse still increases to $\sim 500 \mathrm{fs}$ ( $2 \mathrm{~m}$ fiber) and $\sim 300$ fs ( $1 \mathrm{~m}$ fiber).

Fig. 7 can also be used to estimate tolerances for photodarkening. For example, if we consider a doubling of the pulse duration for the single-channel case and a reduction of the combining efficiency to $90 \%$ for the CBC system as upper limits on system degradation, then for both criteria the 2-m fiber fails already for less than 2-dB PD while the 1.5-m fiber can tolerate more than $5 \mathrm{~dB}$.

\section{DISCUSSION}

The compression is fixed, optimized for the PD-free fiber, but it may be better to optimize it for the average level of PD. We found that this typically halved the worst-case degradation. However, while the benefits of shortening the fiber to allow for some PD at the expense of a small power penalty seem clear, uncertainties in the PD that a fiber will experience make modifications of the settings of a fixed compressor, which degrade the beginning-of-life performance, more dubious. Note also that whereas we have chosen keep the energy constant, it may well be better to adjust the pump power to minimize the increase in the pulse duration for some applications.

The physics of PD is not fully established even in the simpler continuous-wave regime, and the uncertainties are larger still for CPA systems. There is a wide range of pulse parameters, which affect the PD, and which in turn can be expected to evolve with time in different ways in different parts of the fiber. In our configuration, the $\mathrm{Yb}$ excitation level is higher at the input end of the fiber, and this is known to accelerate PD [6]. PD in the signal input end leads to less PD heating, since the signal energy is relatively weak there, On the other hand, also a high signal energy accelerates the PD [7], and this effect will be more severe in the output end. The uncertainty in the longitudinal PD-distribution carries over to the phase distortion, and depending on the precise longitudinal distribution, the impact of a certain total PD propagation loss may be either smaller or larger than for our assumption of uniform PD.

A further complication in assessing the impact of photodarkening is the lack of verified accelerated-aging tests in different regimes. Thus, also experiments with accelerated aging will be fraught by uncertainties, which extend to the fast excitation dynamics of the photodarkening. At the same time, it is still possible that the PD, at least to a significant extent, corresponds to centers with PD excitation dynamics slower than the pulse duration. Whereas this would reduce the phase distortions induced by the instantaneous heating, the combined effect of PD and SPM does not depend on the time constants of the PD excitation and thermalization.

There are also more well-known phase effects which we do not consider and are normally neglected in fiber CPA simulations. The Kramers-Kronig relation describes nonthermal changes in the refractive index when a signal pulse deexcites Yb-ions and the gain thus decreases. The refractive index may increase as well as decrease, depending on the host composition [39]. The size of the resulting phase change may be comparable to that for QD-heating and depends on the extracted energy in the same way, and it too is independent of fiber length. However, the change in thermal phase induced by QD as the PD increases was found to be $\sim 0.3 \mathrm{rad}$. Arguably, this is negligible, and so would be the change in the refractive index according to the Kramers-Kronig relation as the increase in $\mathrm{PD}$ causes a pulse to de-excite more $\mathrm{Yb}$-ions in order to maintain $100 \mu \mathrm{J}$ of output energy.

Also thermal effects that depend on the average power are modified by photodarkening. As it comes to the phase, at high average power, PD-induced heating increases the refractive index in the core, which in turn reduces the mode size [32], [40]. This increases the SPM. For the 2-m fiber with 5-dB PD loss, the path-averaged mode area is reduced by $10 \%$ when the average signal output power is around $0.4-0.5 \mathrm{~kW}$, but the effect is negligible in the case we consider. High average power can also lead to thermal mode instabilities [14]. However, we have not analyzed the TMI regime, which seems incompatible with high-fidelity pulse amplification. 
Although photodarkening has received significant attention in recent years, this has been concentrated to high-averagepower and low-cost systems in which the cost of the pump lasers is a significant factor. This makes them sensitive to even small amounts of PD. By contrast, in femtosecond systems, the cost of the pump lasers is relatively small, e.g., $\$ 1000$ for $50 \mathrm{~W}$, which makes it relatively cheap to compensate for any loss in power caused by $\mathrm{PD}$. In our case, the 5-dB of PD propagation loss induced a pump power penalty of approximately $3 \mathrm{~dB}(5$ W) which, is readily handled by increasing the drive current of a standard pump laser diode. By contrast, although it is also possible to use adaptive phase controllers to compensate for phase distortions, this is much more difficult and normally not used in commercial systems. In this respect, phase distortion is a much more important effect than the power loss in the fiber CPA systems we consider. There is also a tradeoff between PD and fiber length. Even if $5 \mathrm{~dB}$ PD propagation loss appears high, the resulting pulse quality degradation with optimized fiber length may still be acceptable, and then suggests that higher $\mathrm{Yb}$ concentrations in shorter fibers with less SPM may be a better choice, even if the PD loss is much higher than would be acceptable in high-average-power and low-cost systems.

Although our CBC analysis is based on combination of two channels, the analysis can be scaled to $N$ channels. For example, the combining efficiency for $N$ channels may be written as $\eta \approx$ $2 / N-1+2(1-1 / N) \eta_{12} \approx 1-2\left(1-\eta_{12}\right)$ where $\eta_{12}$ is the average combining efficiency between two channels. The second approximation is valid for $N \gg 1$ and shows that the large-count combination efficiency degrades twice as fast as the two-channel combination efficiency. In Fig. 7, the two-channel combination efficiency degrades approximately linearly with PD. In the multichannel system, if we assume that the distribution of total $\mathrm{PD}$ propagation-loss of a channel is uniform on the interval $[0 \mathrm{~dB}, 5 \mathrm{~dB}]$ then the average PD-loss difference between two channels becomes $5 / 3 \mathrm{~dB}$. This then results in a degradation similar to that of the two-channel system with 10/3 $\mathrm{dB}$ PD propagation loss (in one channel).

\section{SUMMARY}

In summary, we used numerical simulations to investigate how photodarkening degrades the quality and combination efficiency of femtosecond pulses with Gaussian spectra in single-channel and coherently combined dual-channel cladding-pumped YDF CPA systems without adaptive control of the spectral phase. Systems with fiber length optimized for the beginning-of-life (without PD) degrade rapidly with the onset of PD, as a consequence of intra-pulse phase distortions. Thus, even if the pump power increases from $5.2 \mathrm{~W}$ to $6 \mathrm{~W}$ to maintain $100 \mu \mathrm{J}$ of output energy in a single-channel system, the compressed peak power drops by $30 \%$ already for $1 \mathrm{~dB}$ of total PD propagation loss. By contrast, a shorter fiber can tolerate $5 \mathrm{~dB}$ of PD loss with $35 \%$ drop of peak power, even at $\mathrm{B}$-integrals as high as $13 \mathrm{rad}$. Beam-combined systems show comparable improvements. We conclude that phase distortions can be a more significant effect of PD than degraded conversion efficiency, and is also more difficult to compensate for. However, a suitable choice of length provides effective mitigation even at $5 \mathrm{~dB}$ of photodarkening, and possibly even higher. Although not considered here, we also note that the fiber drivers of a particle accelerator may also be subject to ionizing radiation, which can also increase the loss [31] and thus the excess heat. This too leads to phase distortions, which we expect can be reduced by designing the fiber for expected end of life conditions.

There are uncertainties in the growth, distribution and fast dynamics of photodarkening, which can all affect our results, and we hope that our study will encourage further investigations of relevant aspects of photodarkening for the type of systems we have considered. In addition, also the operating conditions such as pulse profile and pump configuration will affect the results. For example, a stretched pulse with broader spectrum, $12.8 \mathrm{~nm}$ FWHM (107 fs transform-limited pulse duration), but otherwise the same parameters as the 7.8-nm FWHM pulses we have studied, will experience larger phase distortion. The maximum phase change in this case for 2-m fiber is $10 \mathrm{rad}$, compared to $7 \mathrm{rad}$ for the $7.8 \mathrm{~nm}$ stretched pulse. Therefore, a detailed analysis, ideally backed by accurate PD measurements, will be helpful for the determination of best fiber length. We believe our results are relevant also for high average powers but may be particularly interesting for low powers and systems with high demands on reliability.

\section{ACKNOWLEDGMENT}

We thank Dr Jonathan Price, Prof James R. Leger, and Dr Rüdiger Paschotta for helpful suggestions on the manuscript, and Dr Paschotta for help with RP Fiber Power.

\section{REFERENCES}

[1] J. J. Koponen, M. J. Söderlund, S. K. T. Tammela, and H. Po, "Photodarkening in ytterbium-doped silica fibers," in Proc. SPIE, 2005, vol. 5990, p. 599008.

[2] R. Paschotta, J. Nilsson, P. R. Barber, J. E. Caplen, A. C. Tropper, and D. C. Hanna, "Lifetime quenching in Yb-doped fibres," Opt. Commun., vol. 136, no. 5-6, pp. 375-378, 1997.

[3] K. E. Mattsson, "Photo darkening of ytterbium fiber lasers and amplifiers," Lasers Electro-Optics Quantum Electron. Laser Sci. Conf. (QELS), 2010 Conf., pp. 26-27, 2010.

[4] S. Yoo et al., "Photodarkening in Yb-doped aluminosilicate fibers induced by $488 \mathrm{~nm}$ irradiation," Opt. Lett., vol. 32, no. 12, p. 1626, Jun. 2007.

[5] C. Ye, J. J. Montiel i Ponsoda, A. Tervonen, and S. Honkanen, "Refractive index change in ytterbium-doped fibers induced by photodarkening and thermal bleaching.," Appl. Opt., vol. 49, no. 30, pp. 5799-805, 2010.

[6] S. Jetschke, S. Unger, U. Röpke, and J. Kirchhof, "Photodarkening in $\mathrm{Yb}$ doped fibers: experimental evidence of equilibrium states depending on the pump power," Opt. Express, vol. 15, no. 22, pp. 14838-14843, 2007.

[7] K. E. Mattsson and J. Broeng, "Photo darkening of ytterbium cw fiber lasers," in Proc. SPIE, 2009, no. February 2009, p. 71950 V.

[8] H. Li et al., "Pump Wavelength Dependence of Photodarkening in Yb-Doped Fibers," J. Light. Technol., vol. 35, no. 13, pp. 2535-2540, Jul. 2017.

[9] A. Jolly, C. Vinçont, C. Pierre, and J. Boullet, "Modelling the competition between photo-darkening and photo-bleaching effects in high-power ytterbium-doped fibre amplifiers," Appl. Phys. B, vol. 123, no. 8, p. 227, Aug. 2017.

[10] S. Jetschke, A. Schwuchow, S. Unger, M. Leich, M. Jäger, and J. Kirchhof, "Deactivation of $\mathrm{Yb}^{3+}$ ions due to photodarkening," Opt. Mater. Express, vol. 3, no. 4, p. 452, 2013.

[11] R. Piccoli, D. Mechin, T. Robin, and S. Taccheo, "Lifetime reduction 
due to photodarkening phenomenon in ytterbium-doped fibers and rate equation term.," Opt. Lett., vol. 38, no. 21, pp. 4370-3, 2013.

Changgeng Ye, L. Petit, J. J. Koponen, I-Ning Hu, and A. Galvanauskas, "Short-Term and Long-Term Stability in YtterbiumDoped High-Power Fiber Lasers and Amplifiers," IEEE J. Sel. Top. Quantum Electron., vol. 20, no. 5, pp. 188-199, Sep. 2014.

[13] H.-J. Otto, N. Modsching, C. Jauregui, J. Limpert, and A. Tünnermann, "Impact of photodarkening on the mode instability threshold," Opt. Express, vol. 23, no. 12, pp. 15265-15277, 2015.

[14] A. V Smith and J. J. Smith, "Mode instability in high power fiber amplifiers.," Opt. Express, vol. 19, no. 11, pp. 10180-10192, 2011.

[15] T. Y. Fan, "Laser beam combining for high power, high-radiance sources," IEEE J. Sel. Top. Quantum Electron., vol. 11, no. 3, pp. 567-577, 2005.

[16] A. Klenke et al., "22 GW peak-power fiber chirped-pulseamplification system," Opt. Lett., vol. 39, no. 24, p. 6875, Dec. 2014.

[17] H. Pei, J. Ruppe, S. Chen, M. Sheikhsofla, J. Nees, and A Galvanauskas, "Multi-mJ Ultrashort Pulse Coherent Pulse Stacking Amplification in a Yb-doped $85 \mu \mathrm{m}$ CCC Fiber Based System," in Conference on Lasers and Electro-Optics, 2017, pp. SM1L.2

[18] M. K. Davis, M. J. F. Digonnet, and R. H. Pantell, "Thermal effects in doped fibers," J. Light. Technol., vol. 16, no. 6, pp. 1013-1023, 1998.

[19] M. K. Davis and M. J. F. Digonnet, "Measurements of thermal effects in fibers doped with cobalt and vanadium," J. Light. Technol., vol. 18, no. 2, pp. 161-165, 2000.

[20] L. Dong, "Stimulated thermal Rayleigh scattering in optical fibers," Opt. Express, vol. 21, no. 3, p. 2642, 2013.

[21] Y. Feng, B. M. Zhang, A. V Harish, and J. Nilsson, "Thermally induced distortions of the temporal phase of optical pulses in phosphorous-doped silica fibers," pp. 19-21, 2015.

[22] A. Klenke, E. Seise, J. Limpert, and A. Tünnermann, "Basic considerations on coherent combining of ultrashort laser pulses," Opt. Express, vol. 19, no. 25, p. 25379, 2011.

[23] M. Hanna, F. Guichard, Y. Zaouter, D. N. Papadopoulos, F. Druon, and P. Georges, "Coherent combination of ultrafast fiber amplifiers," J. Phys. B At. Mol. Opt. Phys., vol. 49, no. 6, p. 62004, Mar. 2016.

[24] W. S. Brocklesby, "Progress in high average power ultrafast lasers," Eur. Phys. J. Spec. Top., vol. 224, no. 13, pp. 2529-2543, 2015.

[25] M. Kienel, M. Müller, A. Klenke, J. Limpert, and A. Tünnermann, "12 mJ and $1 \mathrm{~kW}$ Ultrafast Fiber-Laser System using Spatial and Temporal Coherent Pulse Addition," in Lasers Congress 2016 (ASSL, LSC, LAC), 2016, p. AM4A.3.

[26] J. Le Dortz et al., "Highly scalable femtosecond coherent beam combining demonstrated with 19 fibers," Opt. Lett., vol. 42, no. 10, p. 1887, May 2017.

[27] T. Eidam, M. Kienel, A. Klenke, J. Limpert, and A. Tünnermann, "Divided-pulse amplification for terawatt-class fiber lasers," Eur. Phys. J. Spec. Top., vol. 224, no. 13, pp. 2567-2571, 2015.

[28] H. Tünnermann and A. Shirakawa, "Delay line coherent pulse stacking," Opt. Lett., vol. 42, no. 23, p. 4829, 2017.

[29] L. Daniault et al., "Coherent combining of two femtosecond fiber chirped pulse amplifiers," in Advances in Optical Materials, 2011, vol. 36, no. 5, p. AMC2.

[30] Active Fiber Systems, "Active Fiber Systems." [Online]. Available: http://www.afs-jena.de/.

[31] W. S. Brocklesby et al., "ICAN as a new laser paradigm for high energy, high average power femtosecond pulses," Eur. Phys. Journal-Special Top., vol. 223, no. 6, pp. 1189-1195, 2014.

[32] J. W. Dawson et al., "Analysis of the scalability of diffraction-limited fiber lasers and amplifiers to high average power.," Opt. Express, vol. 16, no. 17, pp. 13240-13266, 2008.

[33] R. Paschotta, "Modeling of ultrashort pulse amplification with gain saturation," Opt. Express, vol. 25, no. 16, p. 19112, 2017.

[34] R. Paschotta, "RP Fiber Power." [Online]. Available: https://www.rp-photonics.com/fiberpower.html.

[35] D. C. Brown and H. J. Hoffman, "Thermal, stress, and thermo-optic effects in high average power double-clad silica fiber lasers," IEEE J. Quantum Electron., vol. 37, no. 2, pp. 207-217, 2001.

[36] H. W. H. Lee, "Intra-stark relaxation of $\mathrm{Nd}^{3+}$ in silicate glass: Subpicosecond accumulated photon echo experiments," J. Lumin., vol. 45, no. 1-6, pp. 99-101, Jan. 1990.

[37] E. S. Bliss, "Pulse duration dependence of laser damage mechanisms," Opto-electronics, vol. 3, no. 2, pp. 99-108, May 1971.
"Nanosecond-to-femtosecond laser-induced breakdown in dielectrics," Phys. Rev. B - Condens. Matter Mater. Phys., vol. 53, no. 4, pp. 1749-1761, 1996.

[39] J. W. Arkwright, P. Elango, G. R. Atkins, T. Whitbread, and M. J. F. Digonnet, "Experimental and theoretical analysis of the resonant nonlinearity in Ytterbium-Doped fiber," J. Light. Technol., vol. 16, no. 5, pp. 798-805, 1998.

[40] F. Jansen et al., "Thermally induced waveguide changes in active fibers," Opt. Express, vol. 20, no. 4, p. 3997, Feb. 2012.

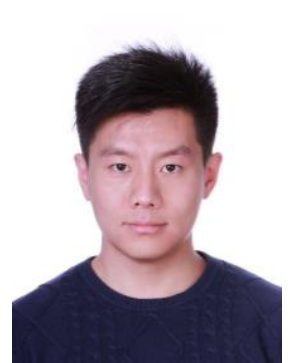

Yujun Feng received the B.Eng. (2012) and the Ph.D. (2017) degree in engineering physics from Tsinghua University, Beijing, China. In 2015, he joined High Power Fiber Laser group at the Optoelectronics Research Centre (ORC), University of Southampton, as a visiting Ph.D. student and is now a research fellow in this group. His research interests focus on high power and ultrafast fiber laser systems.

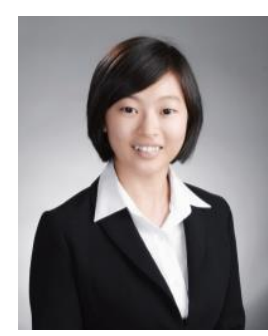

Betty Meng Zhang received the B.E. $\left(1^{\text {st }}\right.$ Honors) and Ph.D. degree in Electrical \& Electronic Engineering from Nanyang Technological University, Singapore.

From 2015 to 2017, she was a Visiting Academic with Optoelectronics Research Centre, University of Southampton, UK. Her research interest includes high energy ultrashort fiber laser systems and special optical fiber designs. Miss Betty Meng Zhang's awards and honors include Best Student Paper Award (ICOCN 2017), Most Outstanding Student Paper Award (OFSIS 2017), Best Student Paper Award (OGC 2016), Women in Photonics Member of the Month (IPS Newsletter), IEEE Photonics Conference 2016 Women in Photonics Student Travel Grant (IPC 2016), IEEE Photonics Society Student Award (IPS).



Johan Nilsson is a Professor at the ORC, University of Southampton, UK, and head of the High Power Fiber Lasers research group. In 1994, he received a doctorate in Engineering Science from the Royal Institute of Technology, Sweden, for research on optical amplification. Since then, he has worked on optical amplifiers and amplification in lightwave systems, optical communications, and guided-wave lasers, first at Samsung Electronics and later at ORC, and has published over 400 scientific articles. He is a fellow of the OSA and the SPIE, and a consultant and co-founder of SPI Lasers. He is a member of the advisory board of the Journal of the Optical Society of Korea and is currently a guest editor for Optics Express. He is a former chair of the Laser Science and Engineering technical group in OSAs Science and Engineering Council and is currently program chair for the EuroPhoton and Advanced Solid State Lasers conferences. 